\title{
A EFETIVIDADE DA TÉCNICA DO MINDFULNESS PARA TRANSTORNO DE ANSIEDADE NA INFÂNCIA: Uma Revisão Sistemática da Literatura
}

\author{
Luana Michele da Silva Alves ${ }^{1}$ \\ Fernanda Marques $\mathrm{Paz}^{2}$
}

Resumo: O presente trabalho teve por objetivo analisar pesquisas com intervenções baseadas em mindfulness para crianças com sintomas de ansiedade, visando à compreensão sobre essas intervenções para o público alvo. Trata-se de uma revisão sistemática da literatura, para a escrita deste artigo foi utilizado o BVS Biblioteca Virtual em Saúde, SCIELO, (Scientific Eletronic Library Online); PEPSIC (Periódicos Eletrônicos em Psicologia), MEDLINE (Sistema Online de Busca e Análise de Literatura Médica) durante os meses de julho, agosto, setembro, outubro e novembro de 2019. Inicialmente foram encontrados 28 artigos, após a verificação dos critérios de inclusão, foi selecionado seis estudos para compor a amostra deste trabalho. A revisão evidenciou que o mindfulness vem se mostrando uma ampla ferramenta podendo ser utilizada como técnica para sintomas ansiosos em crianças. O mindfulness se constitui em um fenômeno complexo, aliado as terapias de terceira onda. As intervenções apresentadas revelaram os efeitos em relação à mindfulness, dentre elas, habilidades de observar conscientemente as suas experiências internas e externas sem distorção e reatividade afetiva, cognitiva ou fisiológica, respondendo adequadamente às situações do seu cotidiano, sendo elas estressantes ou não. Diante disso, os resultados se mostram promissores para o público-alvo, familiares e profissionais que atuam nesta área.

Palavras-chave: Mindfulness, transtorno de ansiedade, infância

\footnotetext{
${ }^{1}$ Acadêmica do Curso de Bacharelado em Psicologia da UNICNEC.

${ }^{2}$ Mestre em saúde coletiva pela UNISINOS.

Perspectiva: Ciência e Saúde,Osório, V. 4 (2): 172-192, Dez 2019.
} 


\title{
EFFECTIVENESS OF MINDFULNESS TECHNIQUE FOR ANXIETY DISORDER IN CHILDREN: A Systematic Literature Review
}

\begin{abstract}
This paper aimed to analyze research with mindfulness-based interventions for children with anxiety symptoms, aiming at understanding these interventions for the target audience. This is a systematic review of the literature, for the writing of this article we used the VHL Virtual Health Library, SCIELO, (Scientific Electronic Library Online); PEPSIC (Electronic Journals in Psychology), MEDLINE (Online Medical Literature Search and Analysis System) during July, August, September, October and November 2019. Initially 28 articles were found, after checking the inclusion criteria, Six studies were selected to compose the sample of this work. The review showed that mindfulness is proving to be a broad tool that can be used as a technique for anxious symptoms in children. Mindfulness is a complex phenomenon, combined with third wave therapies. The interventions presented revealed the effects regarding mindfulness, among them, the ability to consciously observe their internal and external experiences without distortion and affective, cognitive or physiological reactivity, responding appropriately to their daily situations, whether they are stressful or not. Given this, the results are promising for the target audience, family members and professionals who work in this area.
\end{abstract}

Keywords: Mindfulness, Anxiety Disorder, Childhood

\section{INTRODUÇÃO}

A ansiedade é um estado psicológico e fisiológico e caracteriza-se por elementos cognitivos, somáticos, emocionais e comportamentais. Pode tornar-se patológica quando acontece de forma exagerada e sem uma situação real ameaçadora que a desencadeie. Os transtornos de ansiedade mais comuns encontrados em crianças e adolescentes são transtorno ansiedade generalizada, transtorno de ansiedade de separação, fobias específicas e transtorno de estresse pós-traumático (COLON et al, 2017). Dentre os transtornos apresentados a prevalência de transtorno de ansiedade em crianças e adolescentes estima-se em torno de 30\%. Mais de 50\% 
das crianças ansiosas iram experimentar um episódio depressivo como parte de sua síndrome ansiosa (ASBAHR, 2004).

O desenvolvimento emocional das crianças influencia a maneira como elas reagem aos medos e as preocupações, sendo elas normais ou patológicas. Diferentemente dos adultos, as crianças podem apresentar dificuldades em compreender e reconhecer seus medos como exagerados ou irracionais, especialmente as menores, ou seja, quanto menor a capacidade de compreensão de situações que geram medo e/ou desconforto, maior a probabilidade de desenvolver a ansiedade. Portanto a habilidade de auto regulação emocional desempenha um papel fundamental para um melhor ajustamento (THIENGO et al,2014).

A regulação emocional é a capacidade de lidarmos com emoções e experiências que provocam certas reações emocionais. Quando se trata de emoções desagradáveis, é muito comum que tentemos fazer com que essas emoções passem logo. Aquilo que fazemos para "resolver" essas emoções são as estratégias de regulação emocional, que podem ser adaptativas ou desadaptativas (LEAHY et.al., 2013).

A habilidade de regulação emocional pode ser aprendida e compreende a consciência da experiência emocional, o desenvolvimento de um repertório de respostas a ela e sua prática repetitiva em um ambiente seguro. Para isso, são empregadas técnicas que ajudam a regular as emoções de uma maneira mais saudável, assim como o mindfulness e aceitação (BENNINGFIELD et al., 2015).

Mindfulness refere-se à capacidade de prestar atenção ao momento presente, com aceitação e sem julgamento. Tem seu foco na percepção consciente das experiências internas, observando o surgimento de pensamentos e sentimentos, sem fixarem-se aqueles muito valorizados e sem tentar desfazer-se dos negativos. Mindfulness pode ser considerado como uma ferramenta complementar ao tratamento psicoterápico, pois permite ao indivíduo desenvolver capacidade de responder as experiências de uma forma mais equilibrada e menos impulsiva, auxiliando a se desprender da dicotomia, do passado ou futuro (depressão - ansiedade) (GIRARD,FEIX,2016) • Diante deste contexto, as intervenções baseadas em mindfulness apresentam uma forma de melhorar as capacidades auto regulação emocional dos indivíduos. 
Portanto, percebe-se que os problemas de saúde mental na infância, além de prejudicar o desenvolvimento da criança e do adolescente, estão associados ao maior risco de transtornos mentais na vida adulta (GONÇALVES, HELDT, 2009). Em decorrência disso vem se mostrando, cada vez mais importante pesquisar intervenções focadas em prevenir ou atenuar os efeitos da desregulação emocional e cognitiva.

Sendo assim, o objetivo deste trabalho é analisar pesquisas com intervenções baseadas em mindfulness para crianças com sintomas de ansiedade, visando a compreensão sobre essas intervenções para o público alvo, a fim de contribuir para a prática dos profissionais que atuam nessa área.

\section{FUNDAMENTAÇÃO TEÓRICA}

\subsection{Mindfulness}

Conforme descrito por Kabat-Zinn (1990) mindfulness que traduzida para o português chama-se atenção plena, é uma forma de se relacionar com a experiência presente, concentrar-se de maneira intencional e sem julgar o que acontece de momento a momento, exercitando a capacidade de estar atento e consciente a tudo que surgir, internamente e externamente com o presente, além de não estar envolvido com lembranças ou com pensamentos sobre o futuro. Consiste em treinar capacidades de auto regulação e controle atencional, além de ajudar a promover comportamentos pró sociais (RAHAL, 2018; CARUSO, SALOVEY, 2007).

Segundo Menezes e Dell’ Aglio (2009), mindfulness é baseado em exercícios formais e informais. Os formais incluem a varredura mental do corpo e a concentração na respiração, explorando as sensações corporais, com crianças é possível explorar e intervir através dos cinco sentidos. O objetivo é vivenciar a experiência da respiração, dos pensamentos, e outros conteúdos sem querer alterá-los ou controlá-los (REJESKI, 2008; LIMA,OLIVEIRA, GODINHO,2011). Os exercícios informais consistem em vivenciar situações do cotidiano de maneira plena e consciente, com a atenção focada no que está acontecendo, sem julgar ou racionalizar (REJESKI, 2008). 
Roberto Assagioliem (1910), foi o pesquisador pioneiro a integrar conhecimentos da psicologia, budista e Zen, em especial o uso da meditação e atenção plena em um elaborado sistema de psicoterapia chamado psicossíntese. A psicossíntese utiliza-se como abordagem que facilita e aprofunda o autoconhecimento, desenvolvimento pessoal e expressão dos mais elevados talentos e potenciais conscientemente (ASSAGIOLI, 2013).

A atenção plena iniciou a ser pesquisada e integrada com a psicoterapia no início do século XX, em intervenções empiricamente validadas nesta área, surgiram inicialmente no contexto hospitalar com o foco na redução de estresse de profissionais da saúde (KABAT-ZINN, 2003). Atualmente mindfulness tem sido integrada em diversas abordagens psicoterapêuticas, como por exemplo, a Terapia Comportamental Dialética, um programa desenvolvido por Linehan (1987), especificamente para o transtorno de personalidade borderline. O programa mindfulness para redução de estresse (MBR). O MBR consiste em um treinamento grupal, de oito sessões semanais, com o objetivo de vivenciar a respiração, emoções, pensamentos e sensações corporais, sem julgar e deixar se comandar por estes. Entre outras integrações nas terapias de terceira onda, como por exemplo a terapia cognitiva comportamental baseada no mindfulness (MBTC) (KABAT-ZINN, 2013).

No século XXI, consolidou-se a terceira onda de terapias cognitiva com abordagens que utilizam estratégias integrativas, que são todas aquelas que se valem dos pressupostos das terapias cognitivo-comportamentais. Tais estimativas apontam que os processos cognitivos afetam a emoção e consequentemente o comportamento, portanto, pressupõem que, alterando-se as estruturas cognitivas, é possível modificar também as emoções e o comportamento (MELO,2014).

Contudo Friary (2018) ressalta que na Terapia Cognitiva Comportamental tradicional, o terapeuta assume um papel de auxiliar o paciente a modificar as suas crenças e interpretações que induzem a uma visão distorcida da realidade, essa reestruturação do pensamento e cognição tende a ter um efeito na experiência emocional do sujeito, ocasionando uma diminuição de ansiedade e dos sintomas de depressão associados aquela forma de interpretação de dados da realidade. Já na Terapia Cognitiva Baseada em Mindfulness(MBCT), assume que é a relação que temos com os 
nossos pensamentos, nossas emoções e sensações do nosso corpo, que podem aumentar a ansiedade, a depressão e o estresse.

Essa nova técnica, fundamenta-se com foco no contextualismo, enfatizando o papel da consciência plena, da aceitação e compaixão,e neste contexto das terapias de terceira geração o mindfulness tem se destacado por ser uma das técnicas mais estudadas e aplicadas (GUIMARÃES, 2011). A atenção plena vem sendo apresentado como uma alternativa no desenvolvimento de habilidades para lidar com diferentes estados mentais, tais como, ansiedade, depressão, estresse dentre outros.Germer (2016) enfatiza que ao praticar Mindfulness, possibilita às pessoas serem menos reativas diante do que está acontecendo no momento presente e permite a elaboração de uma nova maneira de lidar com a experiência vivenciada.

\subsection{Transtornos ansiosos na Infância}

A ansiedade é um estado psicológico e fisiológico e caracteriza-se por elementos cognitivos, somáticos, emocionais e comportamentais. Podendo tornar-se patológica quando acontece de forma exagerada e sem uma situação real ameaçadora que a desencadeie. Os transtornos de ansiedade mais comuns em crianças incluem o transtorno de ansiedade de separação, transtorno de ansiedade fóbica, transtorno de ansiedade social e transtorno de estresse pós-traumático (COLON et al, 2017).

A ansiedade e o medo passam a ser identificados como patológicos quando são desproporcionais em relação ao estímulo, ou quando interferem na qualidade de vida, bem-estar emocional ou desempenho diário do indivíduo. Uma maneira efetiva de se diferenciar ansiedade típica de ansiedade patológica é basicamente investigar se a reação ansiosa é de curta duração e relacionada ao estímulo do momento ou não. Tais reações exageradas geram um estímulo ansiogênico, devido a associação de diversos fatores hereditários e ambientais, tais como, apego inseguro, temperamento da criança, presença de transtorno de ansiedade nos pais, além da superproteção e comportamentos de evitação na família. (MANFRO \& ASBAHR,2002).

Ansiedade nos pais tem sido apontada como preditor do mesmo transtorno nas crianças. Estilos parentais de super proteção e controle demasiado estão associados à baixa auto eficácia 
em crianças, fator diretamente relacionado aos transtornos de ansiedade. O modelo etiológico dos transtornos de ansiedade pode ser resumido pela interação dos fatores: 1) influências genéticas e ambientais, 2) circuitos neurais envolvidos nas emoções, 3) processos psicológicos, e 4) tendências comportamentais incluindo temperamento (KRAIN et al, 2007; REINECKE et al, 2009; PTERSEN et al, 2010).

Sintomas ansiosos em crianças aparecem com freqüência em outros transtornos psiquiátricos, estes sintomas caracterizam-se por uma ansiedade do transtorno primário (exemplo: medo da separação dos pais numa criança com depressão maior) e não necessariamente constitui um conjunto de sintomas que determina um transtorno ansioso patológico. Mas existem casos em que vários transtornos estão presentes ao mesmo tempo e não se consegue identificar o que é primário e o que não é, ou seja, sintomas primários não são derivados de outras condições psiquiátricas (depressão, transtornos do desenvolvimento, etc.), sendo mais correto afirmar que esse paciente apresenta comorbidades. (CAÍRES, SHINOHARA, 2010). De acordo com o DSM V (2013)o diagnóstico clínico é realizado seguindo os mesmos critérios aplicados aos adultos, com exceção do transtorno de ansiedade de separação que é típico da infância (KRAIN et al, 2007).

Segundo o DSM V (2013), o transtorno de ansiedade de separação é diagnosticado na presença de três (ou mais) dos seguintes sintomas: sofrimento excessivo e recorrente ante a ocorrência ou previsão de afastamento de casa ou de figuras importantes de apego; preocupação persistente e excessiva acerca da possível perda ou de perigos envolvendo figura importante de apego; preocupação persistente e excessiva de que um evento indesejado leve á separação de uma figura importante de apego; relutância ou recusa a sair, afastar-se de casa, ir para a escola, ou qualquer outro lugar, em virtude do medo da separação; temor persistente e excessivo ou relutância em ficar sozinho ou sem as figuras importantes de apego em casa ou em outros contextos; relutância ou recusa em dormir longe de casa ou dormir sem estar próximo a uma figura importante de apego; pesadelos repetitivos envolvendo o tema de separação; repetidas queixas de sintomas somáticos. Os sintomas causam sofrimento clinicamente significativo ou prejuízo no funcionamento social, profissional ou em outras áreas importantes do indivíduo (DSM, 2013). 
Quando as crianças vivenciam a ansiedade de forma intensa, elas podem sofrer diversos prejuízos, como por exemplo, no contexto escolar. A pesquisa de Semple (2005) demonstra que a ansiedade presente nas crianças com idades entre 9 a 12 anos, pode interferir e inibir a capacidade de controlar a atenção, de apreender e absorver informação e, consequentemente, torna mais difícil conseguir superar as dificuldades encontradas no contexto escolar. A autora também ressalta que a ansiedade na infância é um preditor de adolescentes e adultos ansiosos e depressivos, o que também justifica a necessidade de se ter um olhar cuidadoso sobre o tema.

\subsection{Mindfulness e ansiedade}

Nos dias atuais vivenciamos a ansiedade de maneira naturalizada quando antecipamos situações potencialmente ameaçadoras, por exemplo, sentir-se apreensivo, preocupar-se com possíveis resultados negativos e sentir-se no limite e vigilante, no qual essas respostas automáticas podem servir para minimizar algum possível dano ou perda. Entretanto, quando a apreensão ansiosa e a preocupação se tornam recorrente, podemos perder o contato com o momento presente, e a vigilância constante para ameaças futuras pode prejudicar nossa qualidade de vida (GERMER, SIEGEL, FULTON, 2018).

Embora, o medo e a ansiedade sejam respostas inevitáveis e naturais da vida, as mensagens que elas nos enviam podem ser confusas ou distorcidas. Sentimos o impulso de fugir do perigo quando não há ameaça clara presente. Sentimo-nos impulsionados a controlar o futuro, mesmo que em muitos momentos estão além de nosso controle. Portanto, não é surpresa que muitas vezes reagimos ao nosso próprio medo e a nossa ansiedade com julgamento e crítica, encarando nossos pensamentos e emoções como um sinal de fraqueza ou fracasso que nos impedirá de viver uma vida pacífica e gratificante (ROEMER, ORSILIO, 2018).

Germer, Siegel e Fulton (2018), enfatizam que aprender a aceitar as experiências internas que surjam (ex. sensações de pânico, pensamento dos julgamentos dos outros, preocupações sobre o futuro), em vez de tentar supri-las ou evitá-las, pode, paradoxalmente, reduzir o sofrimento e recuperar-se de episódios ansiosos, ou seja a prática de reagir de forma gentil aos nossos sentimentos, emoções e sensações ansiosas, com bondade e compaixão, enquanto damos 
atenção contínua ao momento presente mesmo quando ele repetidamente se desvia em direção a acontecimentos futuros imaginados e temidos, pode ajudar a neutralizar respostas ansiosas aprendidas. Essas estratégias podem nos ajudar a sair do retraimento e da limitação de nossas vidas para vidas mais próximas e plenas. A partir das tradições budistas e comportamentais, as terapias baseadas em mindfulness e aceitação foram desenvolvidas para ajudar essas pessoas a aprender essas novas formas de resposta a fim de reduzir os sintomas de ansiedade e melhorar a qualidade de vida.

Sabemos que o medo e a ansiedade têm função na promoção da sobrevivência de nossa espécie primitiva. Contudo, podemos desenvolver hábitos que nos deixam presos a um ciclo vicioso de evitar situações que não necessariamente é uma ameaça à vida e imaginar catástrofes potenciais sem gerar quaisquer soluções. Seres humanos podem desenvolver hábitos mentais rígidos, respondendo de forma consistente com certos tipos de pensamentos e acreditando neles (devido, em parte, a sua repetição contínua). Quanto mais nossas mentes viajam por esses caminhos, mais desgastados eles se tornam, de modo que nossos pensamentos caem cada vez mais dentro de padrões familiares. Com frequência, esses pensamentos habituais carregam sugestões para evitar alguma possível ameaça, mas seguir essas sugestões pode nos manter mais presos do que seguros(ROEMER, ORSILIO, 2018).

Se respostas internas e comportamentais habituais a ansiedade interferem na capacidade de viver uma vida significativa e gratificante, pode se libertar desses padrões mudando a relação do indivíduo com a ansiedade. Como por exemplo: expandir a percepção da experiência, perceber as reações pelo que elas são e permitir que elas sejam, desenvolver bondade e compaixão para consigo mesmo e escolher de forma intencional envolver-se em atividades que importam para si. Essas são algumas alternativas de resposta que podem ser cultivadas por meio da prática de mindfulness (ROEMER, ORSILIO, 2018).

$\mathrm{O}$ objetivo do tratamento em mindfulness se concentra em ajudar as pessoas a desenvolver uma nova relação com suas experiências internas, que elas sejam ampla em vez de estreita, compassiva em vez de crítica e julgadora e descentrada, ou seja, perceber os pensamentos de forma objetiva e não como verdades globais, em vez de emaranhada e a envolver-se em ações importantes para si, em vez de habitualmente evitá-las. Portanto, integrar 
essa forma de meditação a estratégias de cognitivo-comportamentais pode ser um antídoto para ansiedade crônica e habitual(GERMER, SIEGEL, FULTON,2018).

Também pode ser de grande valia ensinar as pessoas sobre a função das emoções. É compreensível que pessoas com transtorno de ansiedade passem a ver as respostas emocionais como problemáticas. Contudo, emoções primárias, como tristeza, raiva e medo, oferecem informações importantes. Com frequência, ansiedade ou preocupação ocorrem quando outras emoções primárias estão presentes, mas estão sendo evitadas ou ignoradas. Aprender a perceber nossas experiências emocionais pode nos dar acesso às informações que as emoções primárias oferecem. Entretanto as emoções as vezes comunicam informações distorcidas ou incorretas. Ensinar os pacientes a diferenciar emoções claras, que ocorrem em resposta direta à situação em questão, tal como sentir-se triste após uma perda, de emoções turvas, que são distorcidas de alguma forma e oferecem informação menos clara. Sugerimos que as respostas emocionais que são confusas, excepcionalmente duradouras e que aparecem mais intensas do que a situação justifica podem ser turvas(ROEMER, ORSILIO, 2018).

As emoções podem se tornar turvas porque: a) não estamos cuidando bem de nós mesmos (ex. sono ruim, alimentação ruim, sedentarismo); b) estamos preocupados com o futuro ou ruminando sobre o passado, em vez de responder ao momento presente, ou c) estamos reagindo às emoções com julgamento e/ou tentativas de evitá-las. A prática de mindfulness pode nos ajudar a perceber quando nossas emoções são turvas e também a esclarecer o que estamos sentindo. Então, desenvolver habilidades de mindfulness, prestar atenção a qualquer situação, perceber respostas emocionais e reduzir a reatividade, podem naturalmente levar as pessoas a engajar-se de forma mais plena em suas vidas, visto que a reatividade e a esquiva habitual estavam reduzindo esse engajamento (ROEMER, ORSILIO, 2018).

\subsection{Mindfulness para crianças}

O mindfulness na infância está se tornando cada vez mais frequente, ao praticar uma atenção suave no presente momento, as crianças aprendem a responder ao mundo e aos seus próprios desejos e urgências com menos pressa, para que possam perceber suas necessidades e 
seus recursos. Essa consciência e aumento de pausa, convida as crianças e pais a saírem do modo automático de viver. Com habilidades de mindfulness afinadas, adultos e crianças podem identificar melhor seus impulsos e aprender a observar mais de perto as consequências de suas ações no mundo, em si mesmos e nas pessoas a sua volta (FRIARY, 2018)

A atenção plena para crianças oferece uma série de possibilidades como exercitar a empatia, a calma e a gestão emocional desde a infância. Semple (2005) defende que ao praticarem mindfulness, as crianças aprimoram a habilidade de controlar sua atenção e emoção, e, como resultado, conseguem melhorar a concentração e a aprendizagem à medida que desenvolvem uma relação mais adaptativa com as preocupações e as tensões físicas e contribui na redução da ruminação ansiosa.

Ao trabalhar atenção plena com crianças se desenvolve um envolvimento com os pais e a relação familiar. Goodman (2016) esclarece que o desenvolvimento de uma criança evidencia conexões com as emoções dos pais. Semple (2005) também reforça, que há relação entre os sintomas apresentados pelas crianças e o seu ambiente familiar, além disso, o envolvimento familiar durante as intervenções é importante e capaz de promover melhoras significativas na redução de comportamentos desadaptativos apresentados pelas crianças.

As Intervenções Baseadas em mindfulness (IBMs) têm se revelado promissoras para auxiliar as crianças com sintomas de ansiedade. Essas intervenções visam (1) auxiliar as crianças a promover o autoconhecimento, (2) desenvolver a auto regulação emocional, (3) compreender melhor a inter-relação entre pensamentos, sentimentos, comportamentos e (4) aprender a lidar com os desafios da vida, sem ter que fugir ou se sentir paralisadas por eles (DINELLI, ASSUMPÇÃO, 2018).

\section{METODOLOGIA}

Este estudo trata-se de uma revisão sistemática, em que se procurou artigos, capítulos de livros, dissertações e teses em bases de dados, através da seguinte questão de pesquisa: como o mindfulness pode auxiliar no tratamento de crianças com ansiedade? Para a escrita deste artigo foi utilizado o BVS Biblioteca Virtual em Saúde, SCIELO, (Scientific Eletronic Library Online); 
PEPSIC (Periódicos Eletrônicos em Psicologia), MEDLINE (Sistema Online de Busca e Análise de Literatura Médica) durante os meses de julho, agosto, setembro, outubro e novembro de 2019. Foram utilizados os seguintes descritores“mindfulness/ atenção plena", "ansiedade/Anxiety" e "Child/criança", bem como seus equivalentes em português, combinados individualmente através do operador booleano "and". Estes descritores foram devidamente pesquisados nos Descritores em Ciências da Saúde (DeCS). Inicialmente foram encontrados 28 artigos, foram lidos todos os resumos, após esta leitura foram descartados 22 artigos por não entrarem nos critérios de inclusão. Foram excluídos estudos que apresentaram adulto como público-alvo das intervenções. Ressalta-se que alguns artigos contemplaram em uma mesma amostra crianças e adolescentes, entretanto, foram priorizados na análise os resultados das intervenções realizadas com crianças, foram selecionadas pesquisas de 2009 a 2019.

Para compor a amostra deste trabalho são incluídos 6 estudos. Na presente pesquisa foram utilizados apenas os artigos científicos que apresentaram texto completo em português e inglês, em que o periódico estivesse indexado em bases de dados livres. Para exposição dos achados foi feita uma tabela, em que foram descritos os seguintes itens: título da pesquisa, periódico, nome dos autores, ano de publicação, objetivo e resultados.

\section{RESULTADOS E DISCUSSÃO}

A busca inicial resultou em 28 referências. Após a análise da adequação dos textos aos objetivos da revisão, bem como de aplicação dos critérios de inclusão e de exclusão, restaram seis produções para composição do corpus da revisão conforme a Tabela 1. A partir das produções finais foram estabelecidas três categorias temáticas de análise que serão apresentadas na sequência com exemplos de trabalhos recuperados em cada categoria.

Tabela 1 - Descrição dos autores, ano e país de publicação, população, contexto de atendimento e referencial teórico por ordem cronológica 


\begin{tabular}{|c|c|c|c|c|c|}
\hline Cobertt, $\mathrm{M}$. & 2011 & EUA/Florida & Crianças & Escolar & MMI \\
\hline Cicero, $\mathrm{S}$. & 2013 & Nova York & Crianças & Escolar & IBM's \\
\hline Geldert, A. & 2017 & EUA/Minnesota & $\begin{array}{ll}\text { Crianças } & \mathrm{e} \\
\text { adolescentes } & \end{array}$ & Escolar & Calma Yoga e IBM'S \\
\hline $\begin{array}{l}\text { Etherington e } \\
\text { Costello }\end{array}$ & 2018 & Austrália & Crianças & Escolar & $\begin{array}{l}\text { Mindfulness em entre } \\
\text { universais e específica. }\end{array}$ \\
\hline $\begin{array}{ll}\text { Dinelli } & \mathrm{e} \\
\text { Assumpção } & \end{array}$ & 2018 & Brasil / Minas & Crianças & Escolar & Terapias de terceira onda \\
\hline Fortes et.al & 2019 & Brasil/Porto Alegre & Crianças & Clínica & MBCT-C \\
\hline
\end{tabular}

Fonte: Autoria própria, 2019

Gelder (2017) explorou como instrutores de Calma Yoga percebem a yoga e práticas baseadas mindfulness para o alívio de estresse em crianças e adolescentes. A calma Yoga é uma prática que trabalha habilidades que incorpora o movimento, esse movimento permite que as crianças sejam mais conscientes da mente e corpo. A descoberta mais importante deste estudo, foi que após as intervenções as crianças aprenderam a escutar seus corpos, o que é útil, pois o corpo nos dizem o que precisa, e ao ouvir essas necessidades se tornam capazes de ser reguladas, com a ajuda das técnicas e ferramentas no qual são ensinadas para lidar com o estresse, ansiedade e gerir emoções negativas que são experimentados no seu cotidiano.

Já Cicero (2013) analisou o impacto do mindfulness para crianças com ansiedade e suas estratégias de enfrentamento. Os participantes neste estudo mostraram uma diminuição global na ansiedade percebido, redução dos níveis de preocupação e medo, e uma mudança coletiva para estratégias de enfrentamento mais eficazes. Assim Cicero (2013) e Geldert (2017) entenderam que as intervenções baseadas em mindfulness propicia uma ligação da mente e corpo trazendo consciência de suas necessidades. Ao ensinar novas estratégias para lidar com os sintomas apresentados pelo corpo, como, preocupação excessiva e o medo facilitam a compreensão, resultando na diminuição da ansiedade e estresse.

Na revisão sistemática de Dinelli e Assumpção (2018), observou-se que os estudos analisados utilizaram diversos tipos de intervenções baseadas em Mindfulness, sendo elas, Terapia Cognitiva Baseada em Mindfulness (MBCT), Programa de Redução de Estresse Baseado 
em Mindfulness (MBSR), Terapia de Aceitação e Compromisso (ACT) e Terapia Comportamental Dialética (DBT) em crianças e adolescentes entre 5 e 18 anos. Segundo as autoras tais pesquisas avaliadas, indicaram resultados promissores para o público-alvo, familiares e profissionais que atuam nessa área. As intervenções baseadas em mindfulness visam auxiliar as crianças a ampliar o autoconhecimento e o auto cuidado, a regular as emoções e a ajudá-las a vivenciar, de forma saudável, as diversas experiências pelas quais estiverem passando (DINELLI,ASSUMPÇÃO,2018).

Já na revisão de Fortes et. al (2019), foi utilizada somente Terapia Cognitiva Baseada em Mindfulness (MBCT) em crianças e adolescentes de idades entre 9 e 17 anos. Ambas indicaram resultados promissores, demonstrando que as intervenções baseadas em mindfulness são eficazes na diminuição dos sintomas ansiosos, promovendo o autoconhecimento, desenvolvimento da auto-regulação emocional, e contribuição para uma melhor compreensão entre pensamentos, sentimentos e comportamentos, bem como auxilia a lidar com os desafios da vida.

Na pesquisa de Etherington e Costello (2018), objetivou comparar abordagens de entrega direcionada e universal de intervenção de aprendizado social e emocional baseado em mindfulness em crianças com sintomas de ansiedade, afim de comparar qual seria mais eficaz. Programas universais se referem a entregas de intervenções a todos os alunos, independentemente dos sintomas apresentados, já o grupo direcionado se refere a seleção de pessoas devido a sua condição clínica. Os resultados indicaram que a entrega universal é a mais adequada para programas de aprendizagem social e emocional, oferecendo oportunidades para o maior número de estudantes, comparado com grupos clinicamente selecionados. Além disso, foi confirmada que estratégias de mindfulness são componentes úteis na aprendizagem social e emocional, e que elas permitem o compartilhamento de conhecimentos de pessoa para pessoa. Cícero (2013) esclarece que se faz necessário oferecer às crianças, condições para que consigam reconhecer e compreender e se permitir vivenciar as experiências do seu cotidiano em conjunto, a fim de aprender a lidar na interação mesmo que a ansiedade esteja presente.

Apenas a pesquisa de Cobertt (2011) não encontrou resultados positivos, o estudo utilizou uma concepção quase experimental, em grupos, para investigar se as cinco semanas Mindfulness meditação Intervenção (MMI), teria impacto em medidas de atenção, ansiedade, e os níveis de 
cortisol. Os resultados indicaram que aqueles no grupo MMI não diferiram do grupo controle em qualquer das medidas comportamentais.

Em contrapartida, o estudo de Corbett (2011), não relata técnicas utilizadas e também não detalha a adaptação do protocolo adulto para crianças. Apenas esclarece que as sessões de meditação foram de curta duração para as crianças em relação aos adultos, ele também sugeriu que utilizou metáforas ao ensinar técnicas de MMI para as crianças. Além disso, as cinco semanas de intervenção meditação mindfulness (MMI), foram administradas pelos respectivos professores dos participantes. A formação de professores em sessões de informação inicial sobre mindfulness foi ensinada por um colaborador do estudo.

Já no estudo de Fortes et. al (2019), foram descritas algumas técnicas utilizadas em programas baseados em mindfulness na adaptação do protocolo adulto para o infantil, para que se tenha bons resultados, dentre elas estão: atividades sensoriais focadas e simples, meditações de respiração curta, varredura e movimentos corporais conscientes, práticas de visualização e exercícios de desenho ou escrita, práticas experienciais de conscientização consciente sob a forma de atividades e lição de casa. A realização das intervenções feitas pelos seus próprios professores, mesmo que capacitados, também podem ter gerado resultados não significativos comparado aos outros estudos aqui mostrados.

Com relação à descrição das intervenções e seus objetivos, o estudo de Fortes et. al (2019) priorizou programas de terapia cognitiva baseada em Mindfulness para crianças (MBCT-C), sendo que no de Dinelli e Assumpção (2019) abrangeu abordagens de terapias de terceira onda, incluindo o MBCT-C. Com base no MBCT para adultos, foi desenvolvido o MBCT-C, um programa adaptado de 12 sessões para tratar ansiedade em crianças de 9 a 13 anos (Semple et al., 2007). Os artigos de Cicero (2013) e Gelder (2017) e Etherington e Costello (2018) utilizaram intervenções baseadas em mindfulness (IBMs) no geral, sendo que Gelder (2017) integrou as IMBs com a Yoga, mostrando-se eficaz a integração com outras abordagens. Além disso, os autores Etherington e Costello tiveram o enfoque em descobrir qual seria a melhor modalidade de entrega dos programas de habilidades social e emocional. Já a pesquisa de Corbett (2011) não especifica as técnicas utilizadas nas sessões, se referindo a intervenções baseadas em mindfulness e meditação (MMI). 


\section{CONSIDERAÇÕES FINAIS}

A revisão evidenciou que o mindfulness vem se mostrando uma ampla ferramenta podendo ser utilizada como técnica para sintomas ansiosos em crianças. A mindfulness se constitui em um fenômeno complexo, aliado as terapias de terceira onda. As intervenções apresentadas revelaram os efeitos em relação à mindfulness, dentre elas, habilidades de observar conscientemente as suas experiências internas e externas sem distorção e reatividade afetiva, cognitiva ou fisiológica, respondendo adequadamente às situações do seu cotidiano, sendo elas estressantes ou não. Diante disso, os resultados se mostram promissores para o público-alvo, familiares e profissionais que atuam nesta área.

Os trabalhos analisados sugerem que o mindfulness, tem perpassado pela prática de psicólogos e escolares em contextos de intervenção clínica e de atendimento psicossocial. Os contextos escolares e clínico, foram os locais em que esta prática se mostrou com maior eficácia. Nos estudos analisados, algumas limitações foram averiguadas tanto quanto ao método quanto com relação à inclusão de informações pertinentes para compreensão dos trabalhos, sendo elas os protocolos e técnicas utilizadas, contextos, locais, modalidade de intervenções e integrações.

No Brasil a prática de mindfulness é pouco estudada, o que podemos encontrar na literatura internacional são experimentos que mostra o quanto esta prática tornou-se eficaz em contextos escolares, diminuindo assim níveis consideráveis de ansiedade. Nesse sentido aponta-se a necessidade da realização de pesquisas brasileiras na saúde mental infantil atrelando está prática, que visa a oferecer para as crianças um desenvolvimento saudável.

\section{REFERÊNCIAS BIBLIOGRÁFICAS}

ASBAHR, F.Transtornos ansiosos na infância e adolescência: aspectos clínicos e neurobiológicos, Jornal de Pediatria - Vol. 80, N²(Supl), 2004. 
ASSAGIOLI, R. Psicossíntese: as bases da psicologia moderna e transpessoal, São Paulo, Cultrix, 2013

BENNINGFIELD, M. M.; POTTER, M. P.; BOSTIC, J. Q. EducationalImpactsofthe Social andEmotionalBrain. ChildandAdolescentPsychiatricClinicsof North America, 2015.

CAÍRES M. SHINOHARA H.; transtornos de ansiedade na criança: um olhar nas comunidades; Revista brasileira de terapias cognitivas, Rio de Janiero, v. 6, n. 1, p. 62-84,2010.

CARUSO, D.R.; SALOVEY, P. Liderança com inteligência emocional.In: liderando e administrando com competência e eficácia. São Paulo. M. books, 2007.

CICERO, S. The ImpactofMindfulnessonAnxietyandCopingStrategies in Children: A QuantitativeStudy. The CollegeatBrockport: StateUniversityof New York, 2013.

CORBETTI, M. The effectof a mindfulnessmeditationinterventiononattention, affect, anxiety, mindfulness, and salivar cotisol in schoolagedchildren. Florida AtlanticUniversity, Florida, 2011.

CONLON,D. S. B.; MAYNARD, B. R.; BRENDEL, K. E.; FARINA, A. S. J. Mindfulnessbased interventions for youth with anxiety: a systematic review and meta-analysis. American Family Children's Hospital, University of Wisconsin, Madison, WI, USA, P.1-11,2017.

DINELLI L. ASSUMPÇÃO A. intervenções baseadas em mindfulness para crianças com sintomas de ansiedade: Revisão integrativa. Revista da graduação em psicologia da PUC, Minas, v.3 nº, p.67-78, 2018.

DSM-V. Manual diagnóstico e estatístico de transtornos mentais. Porto Alegre: Artmed, 2013. 
ETHERINGTON, V; COSTELlO, S. Comparing Universal andTargeted Delivery of a Mindfulness-BasedProgram for Anxiety in Children. JournalofPsychologistsandCounsellors in Schools, p. 23-38, 2019.

FORTES, P; WILHELM, A; PETERSEN, C; ALMEIDA, R. Mindfulness em crianças com ansiedade e depressão: uma revisão sistemática de ensaios clínicos. Contextos clínicos. v. 2, nº 2 , 2019.

FRIARY, V. Qual o benefício de mindfulness na infância? In:Mindfulnesspara crianças: estratégias da Terapia Cognitiva Baseada em Mindfulness (MBTC).Novo Hamburgo: editora Sinopsys, p.15-16,2018.

FRIARY, V. O que é a terapia cognitiva baseada em mindfulness (MBTC)? In:Mindfulnesspara crianças: estratégias da Terapia Cognitiva Baseada em Mindfulness (MBTC).Novo Hamburgo, editora Sinopsys, p.105-108,2018.

GELDERT, A.Effectsof Yoga andMindfulness-BasedPracticeson Stress andAnxiety in ChildrenandAdolescents.Master of Social WorkClinicalResearchPapers. Universidade de S.T Catherine, 2017.

GERMER, C. K. Mindfulness: O que é? Qual é a sua importância? In: GERMER, C. K.; SIEGEL, R. D.; FULTON, P. R. Mindfuless e psicoterapia. 2. ed. Porto Alegre: Artmed, p. 2-36, 2016.

GERMER, C. K.; SIEGEL, R.; FULTON, P.;Mindfulness: O que é? Qual é a sua importância? In: Mindfuless e psicoterapia. 2. ed. Porto Alegre: Artmed, p. 2-36, 2016.

GIRARD T. FEIX L. mindfulness: concepções teóricas e aplicações clinicas, Revista das ciências da saúde do oeste baiano, Higia, p.94-124. 2016. 
GONÇALVES, D.H.; HELDT, E. Transtorno de ansiedade na infância como preditor de psicopatologia em adultos. Revista gaúcha de enfermagem. Porto Alegre, V.30, n.3, p.533-41,2009.

GOODMAN, T.A. Trabalhar com crianças. In: GERMER, C. K.; SIEGEL, R. D.; FULTON, P. R. Mindfuless e psicoterapia. 2. ed. Porto Alegre: Artmed, p. 247-266, 2016.

GUIMARÃES, S. S. Técnicas Cognitivas e Comportamentais.In: Psicoterapias cognitivo-comportamental um diálogo com a psiquiatria.Porto Alegre: Artmed, 2011.

KBAT-ZINN, J. Using the wisdom of your body and mind to face stress, pain, and illness (2a ed.) New York, Bantam Books, 2013.

KABT-ZINN, J. Mindfulness-based interventions in context: Past, present, and future. Clinicalpsychology: scienceandpractice, 2003.

KRAIN, A.; GHAFFARI, M.; FREEMAN, J.; GARCIA, A.; LEONARD, H.; PINE, D. AnxietyDisorders In Martin A; Volkmar (Eds.). Lewis's ChildandAdolescentPsychiatry: A comprehensiveTextbook. Four Edition. Philadelphia:Linppincot Williams \&Wilnkins, 2007.

LEAHY, R. L.; TIRCH, D.; NAPOLITANO, L. A. Atenção Plena (Mindfulness) IN: Regulação Emocional em Psicoterapia: um guia para o terapeuta cognitivo-comportamental. Porto Alegre: Artmed, p. 111-137, 2013.

LEAHY, R. L.; TIRCH, D.; NAPOLITANO, L. ARegulação Emocional em Psicoterapia: um guia para o terapeuta cognitivo-comportamental. Porto Alegre, Artmed,2013. 
LIMA, M.P.; OLIVEIRA, A.L; GODINHO, P. Promover o bem-estar de idosos institucionalizados: um estudo exploratório com treino de mindfulness. Revista portuguesa de pedagogia, Coimbra, V.45, n.1, p.165-83,2011.

MENEZES, C.B.; DELL'AGLIO, D.D. Porque meditar? A experiência subjetiva da pratica de meditação. Psicologia em estudo, Maringá, V. 48, N.3, P. 135-41,2008.

MELO, W. et al. Estratégias psicoterápicas e a terceira onda em terapia cognitiva. Novo Hamburgo: Sinopsys, 2014.

MANFRO, G. \& ASBAHR, F. R. Estudo retrospectivo da associação entre transtorno de pânico em adultos e transtorno de ansiedade na infância. Revista Bras. Psiquiatria, 2002.

PETERSEN, C.; BUNGE, E.; MANDDIL, J.; GOMAR, M. Terapia Cognitivo-Comportamental para os transtornos de Ansiedade, 2010.

REINECKE, M.; DATTILIO, F.; FREEMAN, A. Terapia Cognitiva com crianças e adolescentes. Relatos de caso e a prática clínica. LPM editora,2009.

ROEMER, L.; ORSILLO, S. M. Ansiedade: aceitar o que vem e fazer o que importa. In: GERMER, C. K.; SIEGEL, R. D.; FULTON, P. R. Mindfuless e psicoterapia. 2. ed. Porto Alegre: Artmed, p. 171-188, 2016.

RAHAL, G. Atenção plena no contexto escolar: benefícios e possibilidades de inserção. Psicol. Esc. Educ., Maringá, v. 22, n. 2, p. 347-358, Aug, 2018.

REJESKI,W. Mindfulness: reconnectingthebodyandthe mind in geratric medicine andgeratology. thegeratologist, Winston-salem, v.48, n.2, p135-41,2008. 
SEMPLE, R. J. Mindfulness-basedcognitivetherapy for Children: A RandomizedGroupPsychotherapyTrialDevelopedtoEnhanceAttentionandReduceAnxiety. 2005. Tese (Doutorado) - Universityof Southern California, 2005.

THIENGO, D; CAVAlCANTE, M; LOVIS, G. Prevalência de transtornos mentais entre crianças e adolescentes e fatores associados: uma revisão sistemáticaJ. bras. psiquiatr. vol.63 n.4, Rio de Janeiro, 2014. 